\title{
Work-relatedness of inguinal hernia: a systematic review including meta-analysis and GRADE
}

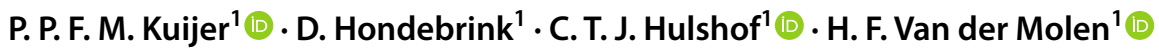

Received: 10 April 2020 / Accepted: 25 May 2020 / Published online: 30 May 2020

(c) The Author(s) 2020

\begin{abstract}
Purpose Clinicians need to know whether inguinal hernia (IH) can be attributed to work to answer questions regarding prevention and medical causation. This review describes whether work-related risk factors are associated with IH.

Methods A systematic review was performed in Medline via PubMed until February 3rd, 2020. Inclusion criteria were that IH was diagnosed by a clinician, and workers exposed to work-related risk factors were compared to workers less exposed or not at all. A quality assessment and a meta-analysis using Cochrane's RevMan 5.3 were performed, including GRADE for quality of evidence.

Results The search resulted in 540 references. Fourteen studies fulfilled the inclusion criteria, of which three were included in a meta-analysis, all three being of high quality, including 621 workers diagnosed with $\mathrm{IH}$. The meta-analysis revealed significant associations with physically demanding work (OR 2.30, 95\% CI 1.56-3.40). Two prospective studies, including 382 and 22,926 cases revealed associations that this was true for male workers with a lateral IH that reported standing or walking for more than six hours per workday (OR 1.45, 95\% CI 1.12-1.88) or lifting cumulative loads of more than $4000 \mathrm{~kg}$ per workday (OR 1.32, 95\% CI 1.27-1.38). The level of certainty for the latter two work-related risk factors was moderate and high according to GRADE.

Conclusion Lateral IH among males is associated with work-related risk factors depending on the level of exposure to the time standing/walking per workday, or the amount of load lifted per workday.
\end{abstract}

Keywords Occupational disease $\cdot$ Risk factors $\cdot$ Occupational exposure $\cdot$ Etiology $\cdot$ Prevention

\section{Introduction}

Worldwide, more than 20 million patients undergo groin hernia repair annually, a vast majority being male workers of working age [1]. The international guidelines for groin hernia management established that risk factors for primary inguinal hernia (IH) among adults include: family history, previous contra-lateral hernia, male sex, age, abnormal

Electronic supplementary material The online version of this article (https://doi.org/10.1007/s10029-020-02236-0) contains supplementary material, which is available to authorized users.

\section{P. P. F. M. Kuijer}

p.p.kuijer@amsterdamumc.nl

1 Netherlands Center for Occupational Diseases, Coronel Institute of Occupational Health, Amsterdam Public Health Research Institute, Amsterdam Movement Sciences, Amsterdam UMC, Location AMC, University of Amsterdam, Amsterdam, The Netherlands collagen metabolism, prostatectomy, and low body mass index [1]. Regarding work, the conclusion was that contradictory evidence existed that social class, occupational factors, and workload affect the risk of IH repair [1-3], and that heavy lifting may predispose to IH formation $[1,4]$. The search for the international guidelines for groin hernia management was conducted until July 2015 [1]. Since then, new prospective cohort studies have been published that might alter the inconclusive evidence regarding the effect of work $[5,6]$. Moreover, no meta-analysis has yet been performed to substantiate the evidence.

This knowledge regarding the work-relatedness of $\mathrm{IH}$ is of importance for patients and clinicians in order to answer questions regarding prevention and medical causation [7, 8]. For prevention, a prerequisite is knowing whether workrelated risk factors actually do matter in the onset or worsening of a disease [9]. In addition, if data allow, clinically relevant exposure threshold limits can be formulated, as is done for several other prevalent diseases like carpal tunnel 
syndrome [10], lateral epicondylitis [11], specific shoulder disorders [12], hip and knee osteoarthritis [13, 14], and lumbosacral radiculopathy syndrome [15]. Regarding medical causation, many countries provide financial compensation when a disease is recognised as an occupational disease, like the Unites States of America, Canada and many countries in the European Union, like Italy, France, and Germany. Therefore, the aim of this systematic review is to assess to what extent work-related risk factors are associated with clinically assessed IH among workers.

\section{Methods}

The systematic review and meta-analysis were performed in line with the criteria of the PRISMA statement [16] (Online Appendix I). No review protocol was published beforehand.

\section{Eligibility criteria}

The following inclusion criteria were used: the study was written in English or German; the study presented original data; participants were workers; IH was diagnosed by a clinician without taking into account the diagnostics tests used; work-related risk factors were described in terms of, for instance, type of industry, job or occupation, physical workload, or specific occupational activities like lifting. To obtain a good overview of the data, all study designs and all follow-up periods were included as long as the data were described in terms of IH being present or not, and exposure was described in terms of exposed versus less exposed or non-exposed.

\section{Search and source}

A systematic literature search was performed in Medline using Pubmed, until February 3rd, 2020. The search strategy involved combining searches for the disease $\mathrm{IH}$, terms for work-related exposure, and for epidemiological studies on risk factors. In addition, the references of the included studies were screened and personal files of the authors for additional studies. Table 1 shows the search strategy.

\section{Study selection}

After duplicates from PubMed were removed, two reviewers (PK, DH) independently checked the fulfilment of the inclusion criteria. We first screened titles and abstracts and excluded studies that did not fulfil the inclusion criteria. Of the remaining references, we obtained the full text and assessed them independently for eligibility based on the full texts. Any disagreements by the two reviewers were resolved through discussion and if necessary, a third assessor (HvdM) made the final decision.

\section{Data collection}

The following data were extracted by one author (DH) and independently checked by a second author (PK): author, year of publication, study design; case definition of IH; source of retrieving participants; number and characteristics of participants like sex and age; exposure definition; number of workers with or without IH for the described exposure categories; (adjusted) risk estimate like Odds Ratios (OR) and 95\% confidence intervals (95\% CI) (Online Appendix II).

\section{Quality assessment}

For the quality assessment of each study, a total of 16 items across five categories were assessed by two reviewers independently (PK, DH) [12]. The five categories were: (1) study population (three items, for instance positive if the participation of both the exposed and unexposed groups was $\geq 70 \%$ ); (2) assessment exposure (three items, for instance whether the exposure was assessed by an independent person and not based on self-report); (3) assessment outcome (three items, for instance whether IH was diagnosed by a clinician); (4) study design (four items, for instance whether the follow-up period was $\geq 1$ year); and (5) data analysis (three items, for instance whether the method used to control for confounding was described). Each item was scored as 'positive', 'negative', or 'unclear'. Differences in outcome were mutually discussed until consensus was reached. High quality was defined as 11 or more items being rated as 'positive' out of a total of 16 criteria [12]. The quality assessment was performed for studies used in
Table 1 Search strategy in Medline using PubMed performed February 3rd, 2020

\begin{tabular}{ll}
\hline & PubMed \\
\hline Worker & $(($ adult[mesh]) OR (adult[tiab]) OR (middle aged[mesh]) OR (middle aged[tiab])) \\
Inguinal hernia & $(($ inguinal hernia[mesh]) OR (inguinal hernia[tiab]) $)$ \\
Work-related etiology & $\begin{array}{l}((\text { occupational disease*[mesh]) OR (occupational disease*[tiab]) OR } \\
(\text { risk factor*[mesh]) OR (risk factor*[tiab]) OR (work-related[tiab]) OR } \\
\end{array}$ \\
& $($ etiology[mesh]) OR (etiology[tiab]) $)$ \\
\hline
\end{tabular}


the meta-analyses regarding physical workload and/or specific occupational activities.

\section{Data analysis}

To answer the research question, a meta-analysis was performed to establish whether risk factors were sufficiently homogeneous across at least two studies. For each risk factor, the highest versus the lowest exposures as reported in the studies were used. We calculated a pooled OR and 95\% CI for each risk factor, including $I^{2}$ as measure of consistency, using a random effects model in Cochrane's RevMan 5.3, for both the high and low risk of bias studies combined and only for the studies with a low risk of bias, if possible. The results are presented as forest plots including the contribution of each study (weight) to the overall effect (Mantel-Haenszel, random) using RevMan 5.3. No additional statistical analyses were performed.

\section{GRADE}

GRADE (Grades of Recommendations, Assessment, Development and Evaluation) was used to assess the quality of evidence for the studies used in the meta-analyses regarding physical workload and/or specific occupational activities. The criteria of the framework for prognostic studies were used [17]. Four levels of quality were used: high, moderate, low, and very low. Therefore, our starting point for the quality of the evidence was 'moderate' for prospective explanatory cohort studies, given the inclusion of only studies specifically focusing on work-related risk factors for IH. Next, the quality of evidence was downgraded based on the following five factors: (1) study strengths (majority of studies having high risk of bias or minority of studies having a prospective study design); (2) consistency $\left(I^{2}>70 \%\right)$; (3) indirectness (a priori not true, given our inclusion criteria that $\mathrm{IH}$ is diagnosed by a physician and the population of interest is workers); (4) imprecision (less than $95 \mathrm{IH}$ patients included or $95 \%$ confidence interval of the effect size of the studied risk factor includes 1 , unless the boundaries of the lower and upper limit of the $95 \%$ confidence interval are smaller than $0.8-1.2$, indicating high certainty of no effect of the studied risk factor for $\mathrm{IH}$ ); and (5) publication bias (Yes). Finally, study findings with effect sizes (i.e. lower limit of $95 \%$ CI Risk estimate $>2.0$ ) and the presence of an exposure-response relationship in the majority of studies (Yes) resulted in an upgrade of the quality of evidence.

\section{Results}

\section{Types of studies and quality}

The search resulted in a total of 540 references, of which 14 fulfilled the inclusion criteria [3, 4, 6, 18-28] (Fig. 1, Online Appendix II). Two studies were case reports [26, 27], one had a cross-sectional design [18], five a case control design [4, 19, 20, 22, 23], one a retrospective cohort design [28] and five a prospective cohort design [3, 6, 21, $24,25]$. Five studies assessed the association between IH and a short-term occupational exposure (single strenuous work-related event) [24-28]. Nine studies assessed the long-term occupational exposure: one study assessed the type of industry and jobs involved [18], six the physical workload [4, 19-23], and two specific occupational activities, namely standing/walking and lifting $[3,6]$ (Online Appendix II). Three of the studies on physical workload were able to be used for the meta-analyses, given sufficient data reported about the presence of IH among exposed versus less exposed workers performing physically demanding work $[4,21,23]$. The three studies in the meta-analyses and two studies on specific occupational activities were all of high quality with scores varying between 12 and 15 , out of a maximum score of 16 (Table 2). The three criteria with the lowest score were: (1) participation rates $<70 \%$; (2) no prospective study design; and (3) insufficient data about completers versus withdrawals.

\section{Physically demanding work}

The meta-analyses based on two high-quality case control studies $[4,23]$ and one high-quality prospective cohort study [21] showed that physically demanding work was associated with an increased risk for IH (OR 2.30, 95\% confidence interval 1.56-3.40) (Fig. 2). Physically demanding work was based on: (1) the self-reported degree of effort of the work categorised into four categories (no, light, medium, and high, and comparing no and light versus medium and high) [23]; (2) a summarised effort score varying between 1 and 10 based on ten interview questions regarding for instance the number of days physically demanding work is performed; the number of times heavy objects are lifted, and whether the work is performed sitting or standing (comparing a score 0-2.5 versus 5.0-10.0) [4]; (3) and a self-report regarding nonrecreational physical activity into three categories (low, moderate, and high) and comparing low versus high [21] (Online Appendix II). 
Fig. 1 Flowchart of the included studies

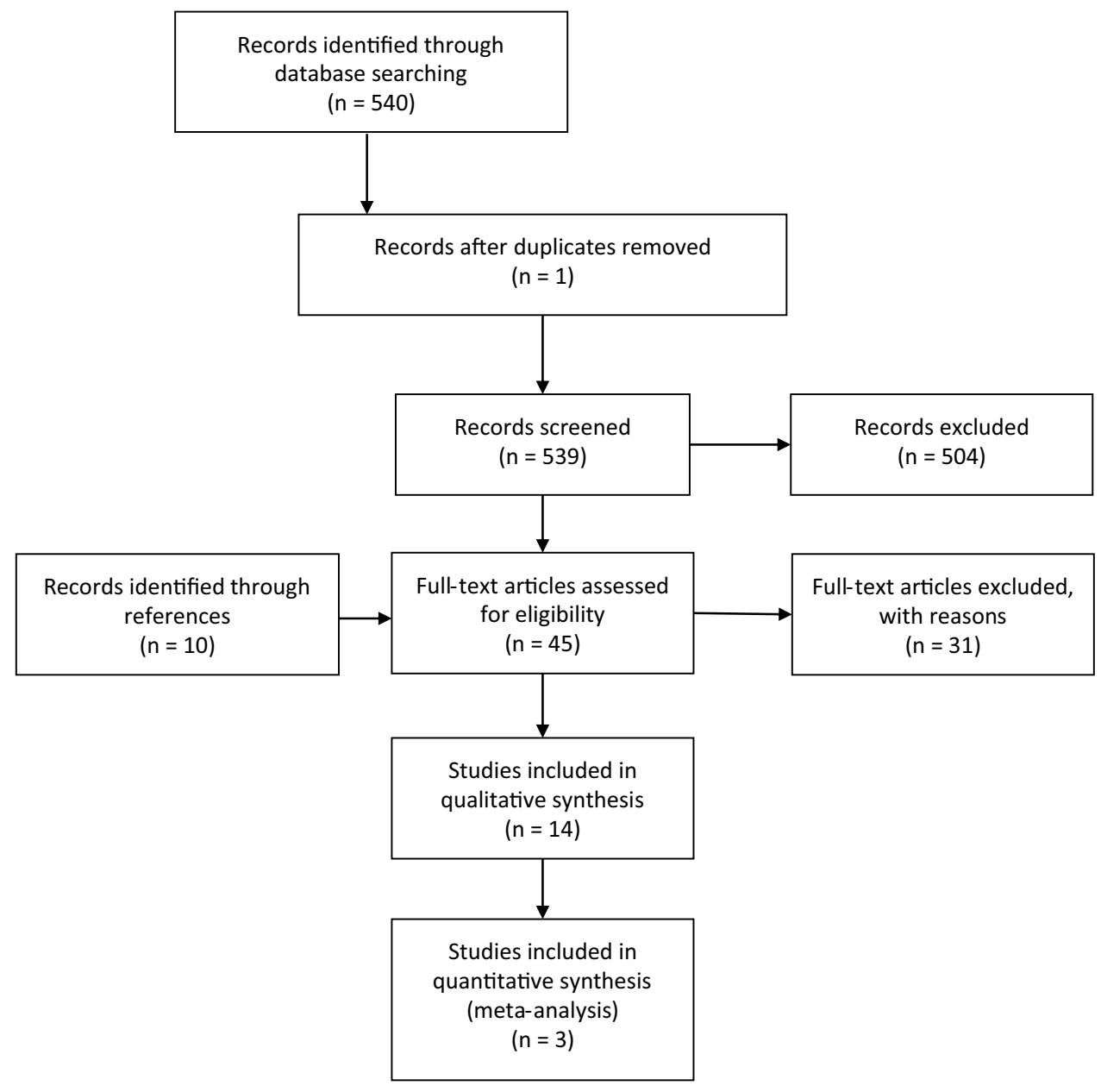

Table 2 Quality assessment of the studies on work-related risk factors graded as positive (+) or negative (-) according to 16 items

\begin{tabular}{|l|c|c|c|c|c|c|c|c|c|c|c|c|c|c|c|c||c|}
\hline \multicolumn{10}{|c|}{ Quality assessment scores } \\
\hline Author & 1 & 2 & 3 & 4 & 5 & 6 & 7 & 8 & 9 & 10 & 11 & 12 & 13 & 14 & 15 & $\begin{array}{l}16 \\
\text { Total } \\
\text { (max 16) }\end{array}$ \\
\hline \hline Carbonell et al. [4] & + & + & + & + & + & + & + & + & + & - & + & + & + & + & + & + & 15 \\
\hline Flich et al. [23] & + & + & + & + & + & + & + & + & - & - & + & + & + & + & + & + & 14 \\
\hline Ruhl and Everhard [21] & + & - & + & - & + & - & + & + & + & + & + & + & - & + & + & + & 12 \\
\hline Vad et al. [3] & + & + & + & + & + & + & + & + & + & + & + & + & - & + & + & + & 15 \\
\hline Vad et al. [6] Total (5) & 5 & 3 & 5 & 4 & 5 & 4 & 5 & 5 & 4 & 3 & 5 & 5 & 3 & 5 & 5 & 5 & + \\
\hline \hline
\end{tabular}

Study population: 1 . Study groups defined, 2. Participation $\geq 70 \%, 3$. Number case $\geq 50$; Assessment of exposure: 4 . Exposure measurement, 5 . Dose-response, 6. Blind for outcome status; Assessment outcome: 7. Outcome definition, 8. Assessment method, 9. Blind for exposure status; Study design: 10. Longitudinal, 11. Inclusion and exclusion criteria, 12. Follow-up period $\geq 1$ year, 13. Info completers versus withdrawals; Data analysis: 14. Data presentation, 15. Consideration of confounders, 16. Control for confounding 


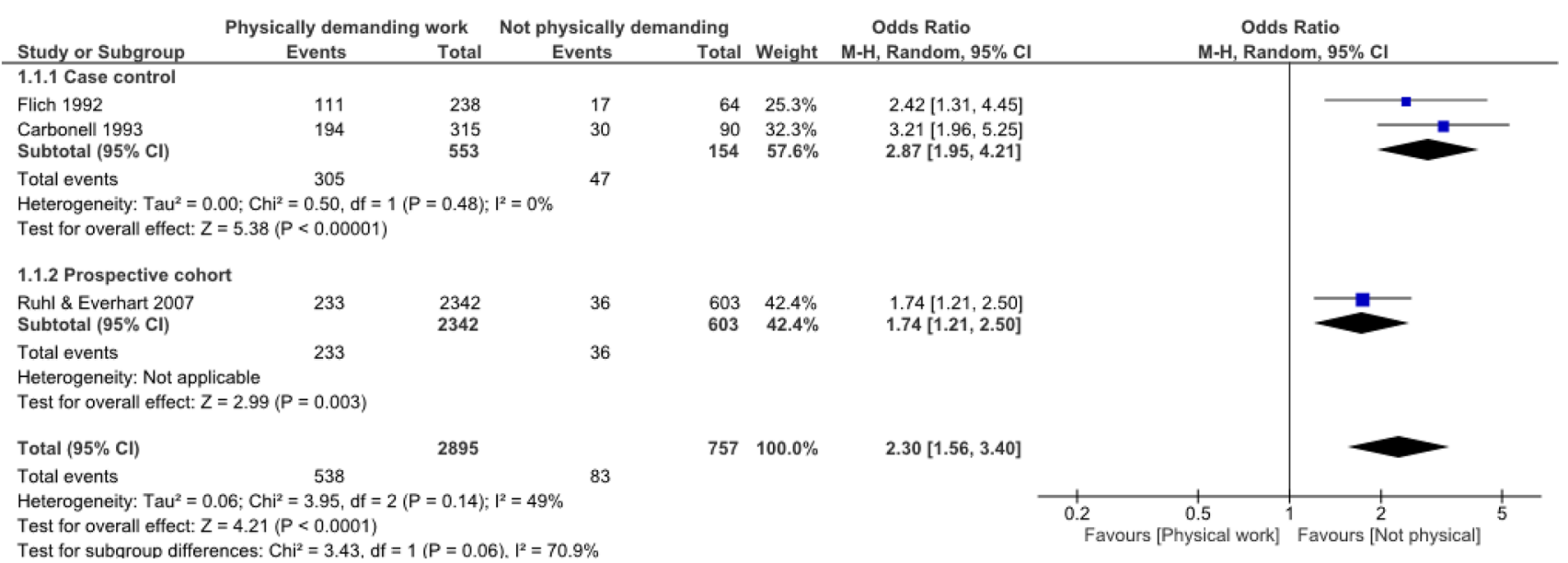

Fig. 2 Forest plot of the association between physically demanding work and IH

\section{Occupational activities}

Vad et al. [3, 6] studied the exposure to the occupational activities of standing/walking and lifting for IH in two prospective cohort studies including 40,395 and $696 \mathrm{IH}$ patients, respectively (Online Appendix II). The exposure to standing/walking and lifting was assessed using information from a Job Exposure Matrix based on expert judgements and year-by-year information on Danish International Standard Classification of Occupations codes for each individual. Due to differences in the definition of exposure categories and potential overlap in similar cases, the data could not be pooled. Their first finding was that these occupational activities were only associated with lateral IH in male workers and not medial IH. Next, standing or walking for more than six hours per workday was associated with $\mathrm{IH}(\mathrm{HR}=1.45$; 95\% CI 1.12-1.88, adjusted for age, body mass index (BMI), leisure time physical activity and smoking, and other factors) and for lifting more than $4000 \mathrm{~kg}$ per workday (OR 1.32; 95\% CI 1.27-1.38, adjusted for age, socioeconomic position and country region).

\section{GRADE}

The evidence for the work-related risk factor of physically demanding work was rated as low (Table 3). The level of evidence was downgraded because two of the three studies had no prospective design, shifting the overall quality from 'moderate' to 'low'. In addition, no upgrades were present. In GRADE terminology, this means: 'Our confidence in the effect estimate is limited. The true effect may be substantially different from the estimated increased risk of 2.30 for IH due to performing physically demanding work'. In contrast, neither of the studies of Vad et al. [3, 6] had a downgrade. Vad et al.
[3] also had one upgrade for the presence of a dose-response relationship [3], thereby shifting the evidence from 'moderate' to 'high'. In GRADE terminology, this means that 'we are moderately confident about the increased risk of 1.45 for a lateral IH due to standing or walking for more than six hours per workday: the true effect is likely to be close to the estimate of the effect, but there is a possibility that it is different'. For lifting, this means that 'we are very confident that the true effect lies close to that of the estimated increased risk of 1.32 for a lateral IH due to lifting more than $4000 \mathrm{~kg}$ per workday'.

\section{Discussion}

\section{Work-related disease}

This review shows that a lateral IH in male workers is associated with physically demanding work that is characterised by standing/walking and lifting. The evidence for both risk factors was of moderate and high quality in GRADE terms, respectively. This review substantiates four of the Hill criteria for causality [29], namely: (1) temporality — the majority of studies had a prospective design; (2) consistency-all five high-quality studies showed that a physical workload was a risk factor [3, 4, 6, 21, 23]; (3) strength-the presence of a dose-response relationship for lifting [3]; and (4) specificity - this appeared true for only a lateral $\mathrm{IH}[3,6]$. To substantiate a fifth criterion for causality, namely plausibility, studies are needed that show that an increased abdominal pressure while standing/walking or lifting results in a protrusion of the abdominal content through the inguinal canal, while sitting may prevent this $[3,6]$. Unfortunately, no papers have been found that studied the effect of prevention at work sites to reduce the incidence of lateral IH among male workers, preferably by reducing the time spent walking/standing 


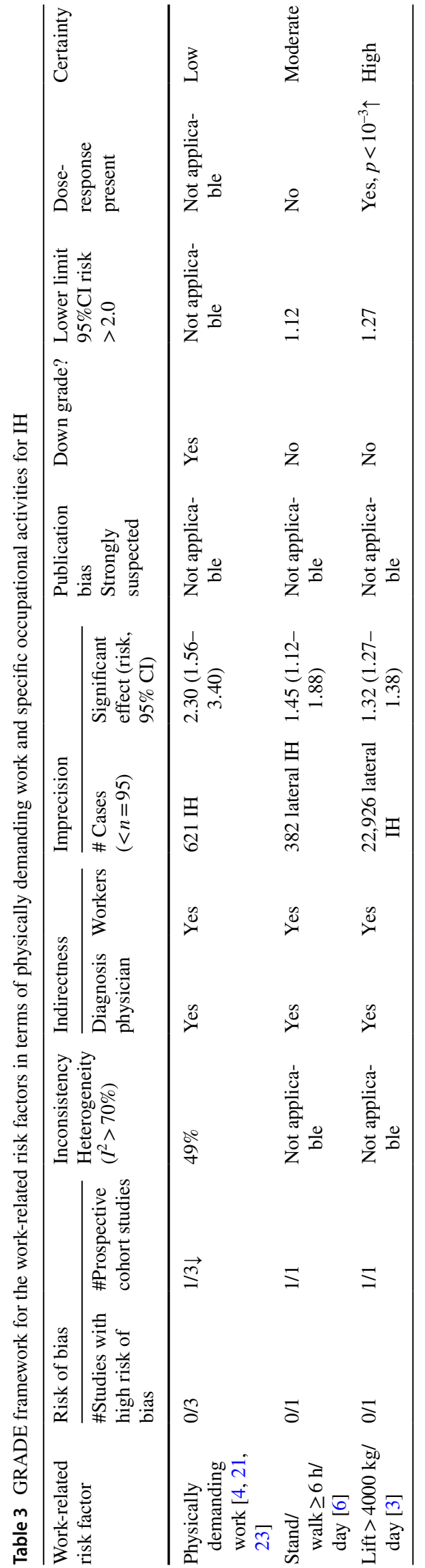

or by reducing the workload due to lifting. If such studies had been performed and indeed had established a preventive effect, this might have substantiated a sixth factor for causality, namely coherence. Based on recently performed cohort studies, a clinically relevant threshold limit for standing/walking might be a maximum of $4 \mathrm{~h}$ per workday, and for lifting a maximum of $1000 \mathrm{~kg}$ per workday [3, 6]. So, based on this review and the above line of reasoning, a future update of the international guidelines for groin hernia management might take into account work as a risk factor for the onset or worsening of lateral IH in males [1].

\section{Strengths and limitations}

A strength of the present review is that the results are based on five high-quality studies including a meta-analysis and the application of GRADE. A second strength of our study is that only clinically assessed IH patients were included. A third strength is that both studies by Vad et al. [3, 6] took into account age as confounder. Moreover, Vad et al. [6] took also into account the personal risk factors BMI, leisure-time physical activity and smoking status, although leisure-time physical activity and smoking status showed no association with lateral (or medial) IH repair in their cohort. This is in line with the reported personal risk factors in international guidelines for groin hernia management [1].

A limitation of our study is that exposures are based on self-reports [4, 21, 23] and expert judgements [3, 6]. Unfortunately, expert judgements and self-reports provide only limited insight into the occurrence of activities [30]. Future studies, preferably on the effectiveness of prevention for lateral $\mathrm{IH}$ among male workers, should gather information from measurements on the time spent walking/standing per workday or data about productivity regarding the amounts of load lifted per workday. Finally, as described in Online Appendix II, the studies by Vad [3, 6] used both data from a similar Danish cohort of first-time inguinal hernia repairs in the period of 1998-2008. To prevent potential overlap due to similar patient data, the two studies were not pooled and for the GRADE assessment only the study with the clearest exposure definition for practice was used.

Author contributions PPFMK, DH, CTJH, HFVdM—study concept and design; PPFMK, DH - acquisition of data; PPFMK, DH-statistical analyses; PPFMK, DH—quality assessments; PPFMK, DH, CTJH, HFVdM-critical revision of the manuscript for important intellectual content and approval of final version; PPFMK, HFVdM-study supervision.

Funding The study is sponsored by the Ministry of Social Affairs and Employment, the Netherlands (Grant no: \#20182019). 
Availability of data and material All data are available and retrievable via PubMed.

\section{Compliance with ethical standards}

Conflict of interest Dr. P. Kuijer reports no disclosures, Mr. Hondebrink reports no disclosures, Prof. Dr. C. Hulshof reports no disclosures, Dr. H. Van der Molen reports no disclosures.

Ethical aprroval Not applicable.

Human and animal rights Not applicable.

Informed consent Not applicable.

Open Access This article is licensed under a Creative Commons Attribution 4.0 International License, which permits use, sharing, adaptation, distribution and reproduction in any medium or format, as long as you give appropriate credit to the original author(s) and the source, provide a link to the Creative Commons licence, and indicate if changes were made. The images or other third party material in this article are included in the article's Creative Commons licence, unless indicated otherwise in a credit line to the material. If material is not included in the article's Creative Commons licence and your intended use is not permitted by statutory regulation or exceeds the permitted use, you will need to obtain permission directly from the copyright holder. To view a copy of this licence, visit http://creativecommons.org/licenses/by/4.0/.

\section{References}

1. The HerniaSurge Group (2018) International guidelines for groin hernia management. Hernia 22:1-165

2. Svendsen SW, Frost P, Vad MV, Andersen JH (2013) Risk and prognosis of inguinal hernia in relation to occupational mechanical exposures - a systematic review of the epidemiologic evidence. Scand J Work Environ Health 39(1):5-26

3. Vad MV, Frost P, Bay-Nielsen M, Svendsen SW (2012) Impact of occupational mechanical exposures on risk of lateral and medial inguinal hernia requiring surgical repair. Occup Environ Med 69(11):802-809

4. Carbonell JF, Sanchez JL, Peris RT, Ivorra JC, Del Baño MJ, Sanchez CS, Arraez JI, Greus PC (1993) Risk factors associated with inguinal hernias: a case control study. Eur J Surg 159(9):481-486

5. Vad MV, Frost P, Svendsen SW (2015) Occupational mechanical exposures and reoperation after first-time inguinal hernia repair: a prognosis study in a male cohort. Hernia 19(6):893-900

6. Vad MV, Frost P, Rosenberg J, Andersen JH, Svendsen SW (2017) Inguinal hernia repair among men in relation to occupational mechanical exposures and lifestyle factors: a longitudinal study. Occup Environ Med 74(11):769-775

7. Boschman JS, Brand T, Frings-Dresen MH, van der Molen HF (2017) Improving the assessment of occupational diseases by occupational physicians. Occup Med (Lond) 67(1):13-19

8. Verbeek J (2012) When work is related to disease, what establishes evidence for a causal relation? Saf Health Work 3(2):110-116

9. Van der Beek AJ, Dennerlein JT, Huysmans MA, Mathiassen SE, Burdorf A, van Mechelen W, van Dieën JH, Frings-Dresen MH, Holtermann A, Janwantanakul P, van der Molen HF, Rempel D, Straker L, Walker-Bone K, Coenen P (2017) A research framework for the development and implementation of interventions preventing work-related musculoskeletal disorders. Scand J Work Environ Health 43(6):526-539

10. Yung M, Dale AM, Kapellusch J, Bao S, Harris-Adamson C, Meyers AR, Hegmann KT, Rempel D, Evanoff BA (2019) Modeling the effect of the 2018 revised $\mathrm{ACGIH}^{\circledR}$ hand activity threshold limit value ${ }^{\circledR}$ (TLV) at reducing risk for carpal tunnel syndrome. J Occup Environ Hyg 30:1-6

11. Descatha A, Albo F, Leclerc A, Carton M, Godeau D, Roquelaure Y, Petit A (2016) Lateral epicondylitis and physical exposure at work? A review of prospective studies and meta-analysis. Arthritis Care Res (Hoboken) 68:1681-1687

12. Van der Molen HF, Foresti C, Daams JG, Frings-Dresen MHW, Kuijer PPFM (2017) Work-related risk factors for specific shoulder disorders: a systematic review and meta-analysis. Occup Environ Med 74(10):745-755

13. Seidler A, Lüben L, Hegewald J, Bolm-Audorff U, Bergmann A, Liebers F, Ramdohr C, Romero Starke K, Freiberg A, Unverzagt S (2018) Dose-response relationship between cumulative physical workload and osteoarthritis of the hip - a meta-analysis applying an external reference population for exposure assignment. BMC Musculoskelet Disord 19(1):182

14. Verbeek J, Mischke C, Robinson R, Ijaz S, Kuijer P, Kievit A, Ojajärvi A, Neuvonen K (2017) Occupational exposure to knee loading and the risk of osteoarthritis of the knee: a systematic review and a dose-response meta-analysis. Saf Health Work 8(2):130-142

15. Kuijer PPFM, Verbeek JH, Seidler A, Ellegast R, Hulshof CTJ, Frings-Dresen MHW, Van der Molen HF (2018) Work-relatedness of lumbosacral radiculopathy syndrome: Review and doseresponse meta-analysis. Neurology 91(12):558-564

16. Moher D, Liberati A, Tetzlaff J, Altman DG, The PRISMA Group (2009) Preferred reporting items for systematic reviews and metaanalyses: the PRISMA statement. BMJ 339:b2535

17. Huguet A, Hayden JA, Stinson J, McGrath PJ, Chambers CT, Tougas ME, Wozney L (2013) Judging the quality of evidence in reviews of prognostic factor research: adapting the GRADE framework. Syst Rev 2:71

18. Kang SK, Burnett CA, Freund E, Sestito J (1999) Hernia: is it a work-related condition? Am J Ind Med 36(6):638-644

19. Lau H, Fang C, Yuen WK, Patil NG (2007) Risk factors for inguinal hernia in adult males: a case-control study. Surgery 141(2):262-266

20. Liem MS, van der Graaf Y, Zwart RC, Geurts I, van Vroonhoven TJ (1997) Risk factors for inguinal hernia in women: a case-control study. The Coala Trial group. Am J Epidemiol 146(9):721-726

21. Ruhl CE, Everhart JE (2007) Risk factors for inguinal hernia among adults in the US population. Am J Epidemiol 165(10):1154-1161

22. Ashindoitiang JA, Ibrahim NA, Akinlolu OO (2012) Risk factors for inguinal hernia in adult male Nigerians: a case control study. Int J Surg 10(7):364-367

23. Flich J, Alfonso JL, Delgado F, Prado MJ, Cortina P (1992) Inguinal hernia and certain risk factors. Eur J Epidemiol 8(2):277-282

24. Pathak S, Poston GJ (2006) It is highly unlikely that the development of an abdominal wall hernia can be attributable to a single strenuous event. Ann R Coll Surg Engl 88(2):168-171

25. Smith GD, Crosby DL, Lewis PA (1996) Inguinal hernia and a single strenuous event. Ann R Coll Surg Engl 78(4):367-368

26. Dickerman RD, Smith A, Stevens QE (2004) Umbilical and bilateral inguinal hernias in a veteran powerlifter: is it a pressureoverload syndrome? Clin J Sport Med 14(2):95-96 (discussion 97)

27. Snyder QC, Kearney PJ (2002) High + Gz induced acute inguinal herniation in an F-16 aircrew member: case report and review. Aviat Space Environ Med 73(1):68-72 
28. Sanjay P, Woodward A (2007) Single strenuous event: does it predispose to inguinal herniation? Hernia 11(6):493-496

29. Hill AB (1965) The environment and disease: association or causation? Proc R Soc Med 58:295-300

30. Van der Beek AJ, Frings-Dresen MH (1998) Assessment of mechanical exposure in ergonomic epidemiology. Occup Environ Med 55(5):291-299
Publisher's Note Springer Nature remains neutral with regard to jurisdictional claims in published maps and institutional affiliations. 